\title{
ORIGINAL
}

\section{Evaluation of various insulin sensitivity indices in lean idiopathic hirsutism patients}

\author{
Sema Ucak, Okcan Basat, Emine Satir and Yuksel Altuntas \\ Division of Endocrinology, Metabolism and Diabetes, Department of Internal Medicine, Sisli Etfal Research and Training \\ Hospital, Istanbul, Turkey
}

\begin{abstract}
Hirsutism is characterized by excessive growth of terminal hair in a male pattern. Idiopathic hirsutism (IH) is a common cause of hirsutism. Since there are few data demonstrating IH is associated with insulin resistance, we tried to assess various insulin sensitivity indices in lean IH and compare with healthy subjects. A cross-sectional study was performed in 71 lean (BMI between 20-25 kg/m²) women (17-39 years old), 31 with $\mathrm{IH}$ and 40 healthy individuals. Blood glucose, insulin, homeostasis model assessment of insulin resistance (HOMA-IR), hepatic insulin sensitivity (ISI HOMA), Quicky index, reciprocal fasting insulin resistance index, fasting Belfiore index, and fasting glucose/insulin ratio (GIR) were estimated using a single fasting sample of glucose and insulin levels. Raynaud indices calculated using the mathematical estimation in a single fasting sample of insulin levels were determined and compared in two groups. Fasting insulin, Raynaud index, HOMA-IR and Fasting insulin resistance index (FIRI) results were higher in IH group than in controls $\left(p<0.01\right.$, for all). Fasting Belfiore index, QUICKI index, ISI HOMA and FIRI ${ }^{-1}$ results were lower in IH group than in controls $(p<0.01$, for all). Our study showed that lean IH patients were more insulin resistant than healthy subjects. We propose that insulin sensitivity indices are useful methods for measuring insulin resistance in IH.
\end{abstract}

Key words: Idiopathic hirsutism, Insulin resistance, Lean patients, Insulin sensitivity indices

HIRSUTISM is characterized by excessive growth of terminal hair in a male pattern. Excessive hair growth in the absence of demonstrable androgen excess in ovulatory women is also referred to as idiopathic hirsutism $(\mathrm{IH})$. IH is diagnosed in women who have hirsutism, normal ovulatory function and normal total or free testosterone levels. IH is a common cause of hirsutism. IH is not associated with any sign of virilization. Its cause is not understood completely. It has been proposed that women with IH have significantly increased cutaneous $5 \alpha$-reductase activity. The presence or absence of such an association is not clear [1].

Polycystic ovary syndrome (PCOS), another cause of hirsutism, is an insulin resistance related syndrome. Even nonobese PCOS individuals are known to have

Submitted Jul. 1, 2011; Accepted Dec. 26, 2011 as EJ11-0101 Released online in J-STAGE as advance publication Feb. 10, 2012 Correspondence to: Sema Ucak, Division of Endocrinology, Metabolism and Diabetes, Department of Internal Medicine, Sisli Etfal Research and Training Hospital, Yenidogan mah. Ersan Sok. No: 834160 Bayrampasa Istanbul, Turkey.

E-mail: seucak@yahoo.com insulin resistance. There are few data demonstrating $\mathrm{IH}$ is associated with insulin resistance. Since the pathogenesis of $\mathrm{IH}$ is still unclear, we tried to assess various insulin sensitivity indices in lean $\mathrm{IH}$ and compare with healthy subjects.

\section{Materials and Methods}

A cross-sectional study was performed in 71 lean (BMI between $20-25 \mathrm{~kg} / \mathrm{m}^{2}$ ) women (17-39 years old), 31 with $\mathrm{IH}$ and 40 healthy individuals. The protocol was approved by the hospital-based ethics committee. The protocol of the study was explained to all of the subjects. Informed consent was obtained before beginning the study.

None of the control subjects had ovarian dysfunction, hirsutism, or hyperandrogenemia. The diagnosis of IH was based on the presence of hirsutism (modified Ferriman-Gallwey score, >8) [2], regular ovulatory menstrual cycles, normal ovaries on ultrasonography and a normal serum androgen profile including free testosterone, and dihydroepiandrosterone sulfate 
Table 1 Formulas and references of indices of insulin sensitivity derived from fasting measurements of glucose and insulin

\begin{tabular}{|c|c|c|}
\hline Insulin sensitivity indices & Formula & Ref. No. \\
\hline Fasting insulin $^{-1}(\mu \mathrm{U} / \mathrm{mL})^{-1}$ & 1 / insulin 0 ' & [3] \\
\hline GIR (U/mg) & Glucose 0 ' / insulin 0' & [4] \\
\hline Raynaud index $\left(\mu \mathrm{IU}^{-1} / \mathrm{L}\right)$ & 40 / insulin 0' & [5] \\
\hline Fasting Belfiore index $\left(\mathrm{pmol}^{-1} \mathrm{~L} / \mathrm{mmolL}\right)$ & $2 /\left[\left(\right.\right.$ insulin $0^{\prime} \times$ glucose $\left.\left.0^{\prime}\right)+1\right]$ & [6] \\
\hline $\mathrm{FIRI}^{-1}\left(\mathrm{mmol}^{-1} / \mathrm{mIUL}\right)$ & $\begin{array}{l}\text { FIRI }=\left(\text { glucose } 0{ }^{\prime} \times \text { insulin } 0\right. \\
\text { FIRI }^{-1}=1 / \text { FIRI }\end{array}$ & [7] \\
\hline QUICKI index $\left(\mu \mathrm{U}^{-1} / \mathrm{mg}\right)$ & 1 / log insulin $0^{\prime}+\log$ glucose $0^{\prime}$ & [8] \\
\hline ISI $_{\text {HOMA }}(\mu U m g)^{-1}$ & $(22.5 \times 18) /$ fasting insulin $\times$ fasting glucose & [5] \\
\hline HOMA- IR $(\mu \mathrm{U}$ mg) & (fasting insulin $\times$ fasting glucose) $/ 22.5$ & [6] \\
\hline
\end{tabular}

0', at 0 minute; GIR, glucose/insulin ratio; FIRI, fasting insulin resistance index; FIRI $^{-1}$, reciprocal fasting insulin resistance index, ISI HOMA, hepatic insulin sensitivity, HOMA-IR, homeostasis model assessment of insulin resistance

Table 2 Age, BMI and hormone levels of patients and control subjects.

\begin{tabular}{lccc}
\hline & Patients $(\mathrm{n}=31)$ & Controls $(\mathrm{n}=40)$ & $p$ \\
\hline Age $($ year) & $27.84 \pm 5.96$ & $27.35 \pm 5.86$ & 0.731 \\
BMI $\left(\mathrm{kg} / \mathrm{m}^{2}\right)$ & $24.90 \pm 2.87$ & $24.95 \pm 1.92$ & 0.923 \\
FSH $(\mathrm{mIU} / \mathrm{mL})$ & $5.32 \pm 0.12$ & $5.24 \pm 0.33$ & 0.751 \\
LH $(\mathrm{mIU} / \mathrm{mL})$ & $7.23 \pm 1,11$ & $7.51 \pm 0.96$ & 0.727 \\
Free testosterone $(\mathrm{pg} / \mathrm{mL})$ & $2.11 \pm 0.12$ & $2 \pm 0.13$ & 0.521 \\
DHEAS $(\mathrm{ng} / \mathrm{mL})$ & $2251.42 \pm 123.41$ & $2214 \pm 103.61$ & 0.734 \\
\hline
\end{tabular}

DHEAS, dihydroepiandrosterone sulfate

\section{(DHEAS).}

Thyroid dysfunction, hyperprolactinemia, nonclassical adrenal hyperplasia, PCOS, and adrenal/ovarian tumors were excluded by appropriate tests and ultrasonography of the ovaries and adrenal glands.

To estimate insulin sensitivity indices we used formulas derived from fasting measurements of glucose and insulin (Table 1).

The insulin sensitivity indices such as homeostasis model assessment of insulin resistance (HOMA-IR), hepatic insulin sensitivity (ISI HOMA), QUICKI index, reciprocal fasting insulin resistance index, fasting Belfiore index, and fasting glucose/insulin ratio (GIR) were estimated using a single fasting sample of glucose and insulin levels. Raynaud index was calculated using the mathematical estimation in a single fasting sample of insulin levels (Table 1).

None of the patients had used any medication known to interfere with carbohydrate metabolism and hirsutism. All subjects were studied in the follicular phase (day 2-9) of their cycles. Ovulation was confirmed by day 24 serum progesterone levels $(>8 \mathrm{nmol} / \mathrm{L})$ in whole group.

Blood glucose levels were studied in whole blood by enzymatic calorimetric methods using commercial devices with an intraassay coefficient variance of $6 \%$ and an interassay coefficient variance of $8 \%$. Plasma insulin levels were studied by RIA with double antibodies (DPC, Los Angeles, CA, USA) with an intraassay coefficient variance of $5.8 \%$ and an interassay coefficient variance of $7.8 \%$.

Hormone measurements were taken after an overnight fast in the the first week of the menstrual cycle. We studied LH and FSH by immunoradiomorphometric assay (IRMA) (Buk-Sangtec Diagnostica GmbH, Germany), free testosterone by RIA (DPC, Los Angeles, CA, USA), and DHEA-S by RIA (Pantex, Santa Monica, CA, USA).

BMI was obtained by dividing the body weight by the square of height $\left(\mathrm{kg} / \mathrm{m}^{2}\right)$.

The results are reported as means $\pm \mathrm{SEM}$. The results of women with $\mathrm{IH}$ and healthy women were compared by using unpaired $t$ test. $P<0.05$ was regarded as statistically significant.

\section{Results}

Mean age and mean body mass index did not differ in patients and controls. No difference was observed in FSH, LH, DHEAS and free testosterone levels between two groups (Table 2). 
Table 3 Insulin sensitivity indices in IH patients and control subjects.

\begin{tabular}{|c|c|c|c|}
\hline & Patients $(n=31)$ & Controls $(n=40)$ & $p$ \\
\hline Fasting insulin $(\mu \mathrm{U} / \mathrm{mL})$ & $18.65 \pm 15.05$ & $9.19 \pm 1.87$ & 0.002 \\
\hline Fasting insulin ${ }^{-1}(\mu \mathrm{U} / \mathrm{mL})^{-1}$ & $0.07 \pm 0.03$ & $0.11 \pm 0.02$ & 0.001 \\
\hline Raynaud index $\left(\mu \mathrm{IU}^{-1} / \mathrm{L}\right)$ & $3.04 \pm 1.40$ & $4.57 \pm 1.16$ & 0.001 \\
\hline Fasting Belfiore index $\left(\mathrm{pmol}^{-1} \mathrm{~L} / \mathrm{mmolL}\right)$ & $0.0041 \pm 0 ., 0019$ & $0.0004 \pm 0.00009$ & 0.001 \\
\hline FIRI & $32.46 \pm 6.26$ & $4.48 \pm 3.47$ & 0.001 \\
\hline $\mathrm{FIRI}^{-1}\left(\mathrm{mmol}^{-1} / \mathrm{mIUL}\right)$ & $0.032 \pm 0.0069$ & $0.31 \pm 0.14$ & 0.001 \\
\hline QUICKI index $\left(\mu \mathrm{U}^{-1} / \mathrm{mg}\right)$ & $0.31 \pm 0.02$ & $0.34 \pm 0.01$ & 0.001 \\
\hline ISI HOMA $(\mu \mathrm{Umg})^{-1}$ & $0.28 \pm 0.13$ & $0.52 \pm 0.10$ & 0.001 \\
\hline HOMA- IR ( $\mu U m g)$ & $4.98 \pm 3.96$ & $1.96 \pm 0.38$ & 0.001 \\
\hline
\end{tabular}

FIRI, fasting insulin resistance index; FIRI-1, reciprocal fasting insulin resistance index; ISI HOMA, hepatic insulin sensitivity; HOMA-IR, homeostasis model assessment of insulin resistance

\section{Fasting insulin}

Fasting insulin levels were significantly higher in $\mathrm{IH}$ group compared to healthy controls $(p=0.002)$ (Table 3 ). When the cut-off point of fasting insulin level for insulin resistance was accepted as $13 \mathrm{mU} / \mathrm{mL}$ according to Ludvig et al. [9], the proportion of insulin resistance was $53 \%$ in IH group.

\section{Raynaud index}

Raynaud index was higher in IH group compared to healthy controls ( $p=0.001$ ) (Table 3 ).

\section{HOMA-IR}

HOMA-IR was significantly higher in IH group than the control subjects ( $p=0.001$ ) (Table 3 ). When the cut-off point of fasting insulin level for insulin resistance was accepted as 3.2 [10] the proportion of insulin resistance was $60 \%$ in IH group and $2.5 \%$ in healthy controls.

\section{FIRI}

FIRI results were higher in IH group than in controls $(p=0.001)$ (Table 3).

\section{Fasting Belfiore index}

Fasting Belfiore index was significantly lower in IH group than the control group ( $p=0.001)$ (Table 3$)$.

\section{QUICKI index}

QUICKI index was significantly lower in IH group than the control group $(p=0.001)$ (Table 3$)$.

\section{ISI HOMA}

ISI HOMA was significantly lower in IH group than the control group ( $p=0.001)$ (Table 3$)$.

\section{FIRI $^{-1}$}

FIRI $^{-1}$ results were lower in IH group than in controls $(p=0.001)$ (Table 3$)$.

\section{Discussion}

Our study showed that IH patients were more insulin resistant than healthy subjects. We propose that insulin sensitivity indices are useful methods for measuring insulin resistance in $\mathrm{IH}$.

IH is diagnosed in women who have hirsutism, normal ovulatory function and normal androgen levels. The diagnosis is one of the exclusion, in which ovulatory dysfunction, elevated circulating testosterone and other causes of androgen excess are ruled out. It occurs more frequently in certain ethnic populations, particularly in women of Mediterranean ancestry [11].

The pathogenesis of IH is not clear. Increased skin $5 \alpha$-reductase activity is thought to be responsible [12]. The relationship of insulin resistance and PCOS is well known [13-18]. Overall, about 50-70\% of women with PCOS have insulin resistance [19].

There are few studies investigating the association of insulin resistance with IH. Unluhizarci et al. [20] showed that IH is associated with some degree of insulin resistance and increased tendency for glucose intolerance notably in obese women with $\mathrm{IH}$.

Sarac et al. [21] found that insulin mediated glucose disposal was lower in normal weight women with $\mathrm{IH}$ than healthy control subjects.

Fattah et al. [22] demonstrated that nonobese IH patients with android obesity based on waist to hip ratio were as insulin resistant as PCOS patients.

Unluhizarci et al. [20] did not choose especially lean patients for their study. BMI was not an exclusion crite- 
rion. Eight patients of 32 were obese and 6 of them had impaired glucose tolerance (IGT). The group was more insulin resistant compared to control subjects. When they adjusted the group for BMI and excluded the IGT subjects, they found that basal insulin and HOMA-IR levels were still higher than the control group.

In our study we especially chose lean women to be able to evaluate insulin resistance independent from obesity.

In our study we found the fact that insulin sensitivity indices such as Fasting insulin, Raynaud index, HOMA-IR and FIRI are higher and Fasting Belfiore index, QUICKI index, ISI HOMA and FIRI ${ }^{-1}$ are lower in IH patients than control subjects supports presence of insulin resistance in those patients.

Based on the cut-off value for HOMA-IR, IH patients were more insulin resistant than controls. Fasting insulin levels were significantly higher in $\mathrm{IH}$ patients than in controls. Based on the cut-off value for fasting insulin, IH patients were more insulin resistant than controls.

It has been suggested that a basal hepatic glucose overproduction defect may be a manifestation of the global insulin resistance in patients at risk for type 2 diabetes mellitus. ISI номA, which reflects basal hepatic glucose production, was lower in IH patients. Altuntas et al. [23] have reported that HOMA-IR should be used as a global insulin resistance test. The data from this study were concordant with their results. We suggest that HOMA-IR is a good predictor of insulin resistance in patients with IH.

Since our group contained only lean women with $\mathrm{IH}$, insulin resistance in $\mathrm{IH}$ patients cannot only be attributed to obesity. We showed that insulin resistance is also a current situation for nonobese $\mathrm{IH}$ patients. The relationship between insulin resistance and IH is not explained well. One explanation for this association might be stimulating effect of insulin/insulin-like growth factor (IGF) system on hair follicle growth [24]. Phillot et al. [25] also demonstrated that insulin/IGF system in vitro has a role on hair follicle growth. In tissues, IGF-1 is produced by mesenchymal type cells and acts in a paracrine and autocrine fashion by binding to the insulin-like growth factor 1 receptor (IGF-1R). This binding activates the receptor tyrosine kinase that triggers the downstream responses and finally stimulates cell division. IGF-1 may therefore be able to stimulate the proliferation of hair follicle cells through cellular signaling pathways of its receptors [26]. Weger et al. demonstrated that IGF-1 signaling is an important mitogenic and morphogenetic regulator in hair follicle biology in IGF-1 transgenic mice [27].

As far as we know, we cannot find any reference indicating that insulin stimulates hair follicle growth. However, hyperinsulinemia leads to increased expression of IGF-1 expression. The liver seems to be the main source providing $75 \%$ of the circulating IGF-1 [28]. The hepatic IGF-1 production is dependent on the signaling of growth hormone (GH) mediated by the growth hormone receptor (GHR). Butler et al. demonstrated that circulating IGF-I concentration increased 4-fold in response to a chronic elevation of plasma insulin in cows. In accordance with the observed increase in plasma IGF-I, hepatic IGF-I mRNA levels were elevated 6.3 -fold. The increase in IGF-I during insulin infusion was associated with an increase in hepatic GHR 1A, a major GHR transcript in bovine liver tissue. They found that GHR 1A was increased 3.6-fold, and that GHR 1A levels were correlated with IGF-I mRNA in response to chronic elevation of plasma insulin [29]. Hyperinsulinemia was also found to stimulate the expression of the GHR in the liver tissue. Thus it is comprehensible that hyperinsulinemia causes an increased production of hepatic IGF-1 by a enhanced signaling of the GHR in the liver. This in turn, raises the amount of circulating IGF-1 and therefore results in cell growth and proliferation [29-31].

In addition both the insulin receptor (IR) and the IGF-IR are transmembrane oligomer receptors which consist of one $\alpha$ - and one $\beta$-subunit. The $\beta$-subunit comprises a tyrosine kinase which undergoes autophosphorylation after the ligand binds to the extracellular $\alpha$-subunit of the receptor. Subsequently insulin receptor substrates and adaptor proteins are recruited, activated and induce two major signaling pathways. On one hand the mitogenic MAPK pathway and on the other hand the metabolic and anti-apoptotic PI3K pathway. The MAPK pathway plays an important role in cell growth and proliferation. The anti-apoptotic influence of the PI3K pathway is mediated through the activation of protein kinase $\mathrm{B}$ which controls among other things apoptosis-regulating transcriptions factors [28]. Cells that express the IR and the IGF-1R may also form a hybrid receptor from two subunits of these two receptors. Insulin manifests a decreased affinity to the IGF-1R and a very low affinity to the hybrid receptors. However IGF-1 retains its high affinity to the IGF-1R as well as to the hybrid receptors. Since there are two subtypes of the IR, the IR-A and the IR-B, there also exist two different hybrid receptors of IGF-1 and IR 
(IGF-1/IR-A, IGF-1/IR-B). As the IR-A homoreceptor, the IGF-1R/IR-A hybrid receptor mainly results in mitogenic signaling. Comparatively the IGF-1R/IR-B hybrid receptor results in metabolic signaling [32, 33]. In summary hyperinsulinemia raises the amount of circulating IGF-1 and shares similar pathways with IGF 1. This similar downstream signaling mechanism of insulin/ IGF-1 system can explain the effect of hyperinsulinemia on hair follicles.

In conclusion, there is a relationship between IH and insulin resistance. This association is also demonstrated for nonobese IH patients. Insulin sensitivity indices seem to be useful in nonobese IH patients for determining insulin resistance.

\section{Limitations}

We did not measure waist to hip ratio in our study group so we cannot know if this relation is because of android obesity.

\section{Conflict of Interest}

No potential conflict of interest relevant to this article was reported.

\section{References}

1. Bulun SE, Adashi YA(2008) The Physiology and pathology of the female reproductive axis. In: Kronenberg HM, Melmed S, Polonsky KS, Larsen PR (eds) Williams textbook of endocrinology. Saunders Elsevier, Philelphia:541-614.

2 Hatch R, Rosenfield RL, Kim MH, Tredway D (1981) Hirsutism: implications, etiology and management. Am J Obstet Gynecol 140:815-830.

3. McAuley KA, Williams SM, Mann JI et al. (2001) Diagnosing insulin resistance in the general population. Diabetes Care 24: 460-464.

4. Hoffman DI, Klove K, Lobo RA (1984) The prevalence and significance of elevated dehydroepiandrosterone sulfate levels in anovulatory women. Fertil Steril 42:76.

5. Raynaud E, Perez-Martin A, Brun J-F, Benhaddad AA, Mercier J (1999) Revised concepts for the estimation of insulin sensitivity from a single sample. Diabetes Care 22:1003.

6. Belfiore F, Iannello S, Volpicelli G (1998) Insulin sensitivity indices calculated from basal and OGTT-induced insulin, glucose, and FFA levels. Mol Genet Metab 63: 134-141.

7. Duncan MH, Singh BM, Wise PH, Carter G, AlaghbandZadeh J (1995) A simple measure of insulin resistance. Lancet 346:120-121.

8. Katz A, Nambi SS, Mather K, et al. (2000) Quantitative insulin sensitivity check index: a simple, accurate method for assessing insulin sensitivity in humans. $J$ Clin Endocrinol Metab 85:2402-2410.

9. Ludvig B, Nolan JJ, Baloga J, et al. (1995) Effect of obesity on insulin resistance in normal subject and patients with NIDDM. Diabetes 44:1121-1125.

10. Marques-Vidal P, Mazoyer E, Bongard V, et al. (2002) Prevalence of insulin resistance syndrome in southwestern France and its relationship with inflammatory and hemostatic markers. Diabetes Care 25:1371-1377.
11. Jiang M, Aittomäki K, Nilsson C, et al. (1998) The frequency of an inactivating point mutation (566C--> T) of the human follicle-stimulating hormone receptor gene in four populations using allele-specific hybridization and time-resolved fluorometry. J Clin Endocrinol Metab 83:4338-4343.

12. Parks JS, Brown MR (1999) Transcription factors regulating pituitary development. Growth Horm IGF Res 9: 8-11.

13. Mandarino L, Baker B, Rizza R, et al. (1984) Infusion of insulin impairs human adipocyte glucose metabolism in vitro without decreasing adipocyte insulin receptor binding. Diabetologia 27:358-363.

14. Burghen GA, Givens JR, KitabchiAK (1980) Correlation of hyperandrogenism with hyperinsulinism in polycystic ovarian disease. J Clin Endocrinol Metab 50:113116.

15. Chang RJ, Nakamura RM, Judd HI, Kaplan SA (1983) Insulin resistance in non-obese patients with polycystic ovarian disease. J Clin Endocrinol Metab 57:356-359.

16. Jialal T, Naiker P, Reddi K, et al. (1987) Evidence for insulin resistance in nonobese patients with polycystic ovarian disease. J Clin Endocrinol Metab 64:10661069.

17. Dunaif A, Segal KR, FutterweitW, Dobriansky A (1989) Profound peripheral insulin resistance independent of obesity, in polycystic ovarian syndrome. Diabetes 38:1165-1174.

18. Toprak S, Yonem A, Cakir B, et al. (2001) Insulin resistance in nonobese patients with polycystic ovary syndrome. Horm Res 55: 65-70.

19. Ovalle F, Azziz R (2002) Insulin resistance, polycystic ovary syndrome, and type 2 diabetes mellitus. Fert Steril 77:1095-1105.

20. Unluhizarci K, Karababa Y, Bayram F, Kelestimur F (2004) The investigation of insulin resistance in patients with idiopathic hirsutism. JCEM 89:2741-2744. 
21. Sarac F, Saygili F, Ozgen G, Tuzun M, Yilmaz C, Kabalak T (2007) Assessment of insulin resistance in idiopathic hirsutism. Gynecol Obstet Invest 63:126131.

22. Abdel Fattah NS, Darwish YW (2009) Is there a role for insulin resistance in nonobese patients with idiopathic hirsutism? Br J Dermatol 160:1011-1015.

23. Altuntas Y, Bilir M, Ozturk B, Gundogdu S (2003) Comparison of various simple insulin sensitivity and beta-cell function indices in lean hyperandrogenemic and normoandrogenemic young hirsute women. Fertil Steril 80:133-142.

24. Itami S, Kurata S, Takayasu S (1995) Androgen induction of follicular epithelial cell growth is mediated via insulin-like growth factor-I from dermal papilla cells. Biochem Biophys Res Commun 212:988-994.

25. Philpott MP, Sanders DA, Kealey T (1994) Effects of insulin and insulin-like growth factors on cultured human hair follicles: IGF-I at physiologic concentrations is an important regulator of hair follicle growth in vitro. J Invest Dermatol 102:857-861.

26. Su HY, Hickford JG, Bickerstaffe R, Palmer BR (1999) Insulin-like growth factor 1 and hair growth. Dermatol Online J 5:1.
27. Weger N, Schlake T (2005) Igf-I signalling controls the hair growth cycle and the differentiation of hair shafts. $J$ Invest Dermatol 125:873-882.

28 Kooijman R (2006) Regulation of apoptosis by insulinlike growth factor (IGF)-I. Cytokine Growth Factor Rev 17:305-23.

29 Butler ST, Marr AL, Pelton SH, Radcliff RP, Lucy MC, Butler WR (2003) Insulin restores GH responsiveness during lactation-induced negative energy balance in dairy cattle: effects on expression of IGF-I and GH receptor 1A. J Endocrinol 176:205-217.

30. LeRoith D, Roberts CTJr (2003) The insulin-like growth factor system and cancer. Cancer Let 195:127-37.

31. Gallagher EJ. et al. (2010) The increased risk of cancer in obesity and type 2 diabetes: potential mechanisms. In: Poretsky L (ed) Principles of Diabetes Mellitus. Springer, US: 2010:583

32. LeRoith D, Werner H, Beitner-Johnson D, Roberts CTJr (1995): Molecular and cellular aspects of the insulinlike growth factor I receptor. Endocr Rev 16:143-163.

33. Yakar S, Pennisi P, Kim CH, et al. D (2005): Studies involving the GH-IGF axis: Lessons from IGF-I and IGF-I receptor gene targeting mouse models. $J$ Endocrinol Invest 28:19-22. 